\title{
A discussion on the existence of the anomalous high and the anomalous low
}

\section{N. Li}

Key Laboratory for Aerosol-Cloud-Precipitation of China Meteorological Administration, School of Atmospheric Physics, Nanjing University of Information Science and Technology, Nanjing, China

Correspondence to: N. Li (shangjineh@163.com)

Received: 6 March 2015 - Revised: 10 August 2015 - Accepted: 17 September 2015 - Published: 5 October 2015

\begin{abstract}
The air flow in a three-way balance between the Coriolis force, the centrifugal force and the pressure gradient force, i.e., the gradient wind, is discussed. The author studies formation mechanisms and possible existence of four types of gradient wind (the normal high, the normal low, the anomalous high and the anomalous low), and proposes reasonable explanation of the evolution of the gradient wind, especially for the anomalous high and the anomalous low, both of which are considered to be pure mathematical solutions and are overlooked in classic literature.
\end{abstract}

Keywords. Atmospheric composition and structure (pressure density and temperature)

\section{Introduction}

Large-scale air flow is quasi-horizontal and frictionless, and it is subjected to Coriolis, centrifugal and horizontal pressure gradient forces. When the Coriolis force (deflection force of Earth rotation), the centrifugal force and the pressure gradient force are in a three-way balance, the air flow is called the gradient wind (Holton, 1992). Since the air moves in a curved path, it is convenient to adopt the natural coordinate to discuss the trajectory. In the natural coordinate, unit vector $\boldsymbol{t}$ is oriented to the direction of the horizontal velocity at each point; unit vector $\boldsymbol{n}$ is vertical to the horizontal velocity and is oriented so as to be positive to the left of the horizontal velocity direction. The gradient wind equation of the balanced flow in natural coordinate can be written as

$\frac{V^{2}}{R}+f V+\frac{1}{\rho} \frac{\partial p}{\partial n}=0$, and the gradient wind speed is

$V_{\mathrm{gr}}=-\frac{f R}{2} \pm\left(\frac{f^{2} R^{2}}{4}-\frac{R}{\rho} \frac{\partial p}{\partial n}\right)^{1 / 2}$

where $V$ is the horizontal velocity of the air, $\rho$ is the density, $\frac{\partial p}{\partial n}$ is the horizontal air pressure gradient along the normal direction, $f=2 \Omega \sin \phi, \Omega$ is the angular velocity of rotation of the Earth, $\phi$ is the geographical latitude and $R$ is the radius of curvature of the air trajectory. The gradient wind is a general motion for large-scale air flow. The geostrophic balance subject to the Coriolis and the pressure gradient force

$f V+\frac{1}{\rho} \frac{\partial p}{\partial n}=0$

with the geostrophic wind speed

$V_{\mathrm{g}}=-\frac{1}{f \rho} \frac{\partial p}{\partial n}$,

is a special case of the gradient balance when $R \rightarrow \infty$. The inertial balance subject to the Coriolis and the centrifugal force

$\frac{V^{2}}{R}+f V=0$,

with the inertial wind speed

$V_{\mathrm{i}}=-f R$,

is a special case of the gradient balance when $\frac{\partial p}{\partial n} \rightarrow 0$.

Because the wind speed in the natural coordinate must be real and nonnegative, it is required that in Eq. (2) the righthand side as a whole is real and nonnegative. In addition, the 


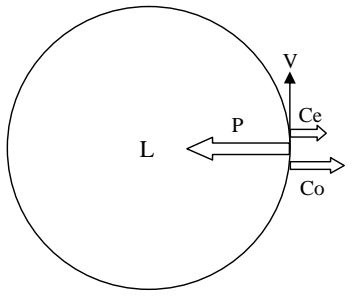

(a)

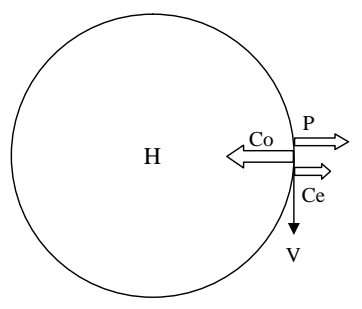

(c)

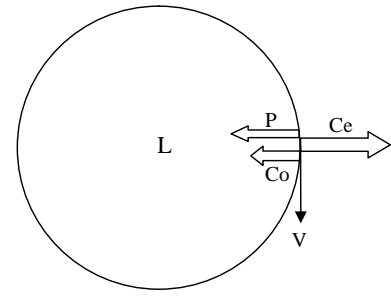

(b)

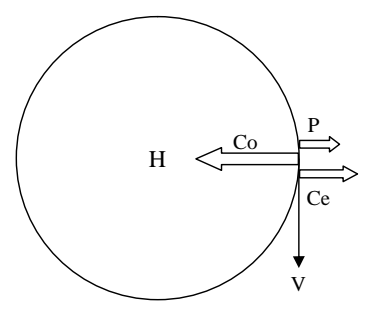

(d)
Figure 1. Force balances for the types of the gradient wind: (a) normal low; (b) anomalous low; (c) normal high; (d) anomalous high. "P" represents the pressure gradient force, "Co" the Coriolis force and "Ce" the centrifugal force.

Table 1. Classification of roots of the gradient wind equation.

\begin{tabular}{lll}
\hline & $R>0$ & $R<0$ \\
\hline$\frac{\partial p}{\partial n}>0$ & $\begin{array}{l}\text { Positive root: unphysical } \\
\text { Negative root: unphysical }\end{array}$ & $\begin{array}{l}\text { Positive root: anomalous low } \\
\text { Negative root: unphysical }\end{array}$ \\
\hline$\frac{\partial p}{\partial n}<0$ & $\begin{array}{l}\text { Positive root: normal low } \\
\text { Negative root: unphysical }\end{array}$ & Positive root: anomalous high \\
\end{tabular}

quantity under the square root should be real and nonnegative. Types of the gradient wind can be discussed according to the signs of $R$ and $\frac{\partial p}{\partial n}$, and are concluded in Fig. 1 and Table 1 as presented by Holton (1992). Types of commonly observed gradient wind are the normal low (Fig. 1a) and the normal high (Fig. 1c), and by contrast it is difficult to observe the anomalous low (Fig. 1b) and the anomalous high (Fig. 1d). It can be seen that the anomalous low refers to the wind direction matching with the anticyclonic flow and that the anomalous high refers to the wind speed that is greater than the normal high.

The gradient wind is a well-known pedagogical concept which can be found in many textbooks on atmospheric sciences. There have been few studies on gradient wind during the past decades. Fultz (1991) derived a nondimensional gradient wind equation divided by the inertial velocity. Through the scope of the dimensionless gradient and geostrophic wind measured in inertial velocity, four types of flow are discussed for different scales. Knox and Ohmann (2006) presented iterative solutions of the gradient wind equation, illustrating characteristics of numerical solutions to full dynamical equations. Willoughby (2011) defined a golden radius for the gradient wind and it is used as a transition mark and calcula- tion criterion between the cyclostrophic, geostrophic and inertial wind. Brill (2014) proposed concepts of contour gradient wind and natural gradient wind to clarify the distinctions when parcel trajectory and its curvature are used in calculation of the gradient wind. Regarding the anomalous gradient wind, previous textbooks have not given clear physical meaning to the anomalous high and the anomalous low, and have generally regarded them as pure mathematical solutions of the gradient wind equation. Some considered them incorrect solutions (Panofsky, 1956; Jacobson, 2005; Wallace and Hobbs, 2006). Some required the solutions in Eq. (2) satisfy $V_{\mathrm{gr}} \rightarrow 0$ when $\frac{\partial p}{\partial n} \rightarrow 0$ as there could be no wind without a pressure gradient (Humphreys, 1940; Godske et al., 1957; Willett and Sanders, 1959; Byers, 1974). Some mentioned that they had not been observed on a large scale but did not give the context (Haltiner and Martin, 1957; Hess, 1959). A few studies attempted to explain the anomalous gradient wind. Alaka (1961) noted that the normal high and the anomalous high meet at the same wind speed when the pressure gradient is at its maximum value. He also proposed a mechanism for the development of the anomalous high. Mogil and Holle (1972) noted that for the normal high, the wind speed is less than twice the geostrophic wind speed, while for the anomalous high, the wind speed is greater than twice the geostrophic value. Based on this criterion, they used two cases in the upper level of the atmosphere to present the possible existence of the anomalous high. Krishnamurti et al. (2005) analyzed the strong wind of jet stream at $200 \mathrm{hPa}$ level south of Japan and found the wind speed is not consistent with the gradient wind of the normal high. They also found the occurrence of the anomalous wind can cause forecast failures for models with constraints of geostrophic or normal gradient wind.

Previous studies did not give comprehensive explanation and possible occurrence for the gradient wind, especially for the two confused anomalous types. In this paper, mechanisms and occurrence of the four types of the gradient wind are studied through theoretical analysis with the aid of a wind-force relation diagram. Section 2 gives an instruction for use of a wind-force relation diagram. Sections 3 and 4 analyze evolution processes and possible occurrence of highpressure gradient wind, and low-pressure gradient wind, respectively. Section 5 gives major conclusions arising from this article and suggests possible directions for future research.

\section{Explanation of a wind-force relation diagram}

In order to better understand the approach used in the discussion, a wind-force relation diagram is presented to analyze evolution of the wind (Fig. 2). The horizontal axis indicates wind speed and the vertical axis indicates magnitude of forces. The curves of wind-force relation are calculated from the force magnitude function of wind speed. 


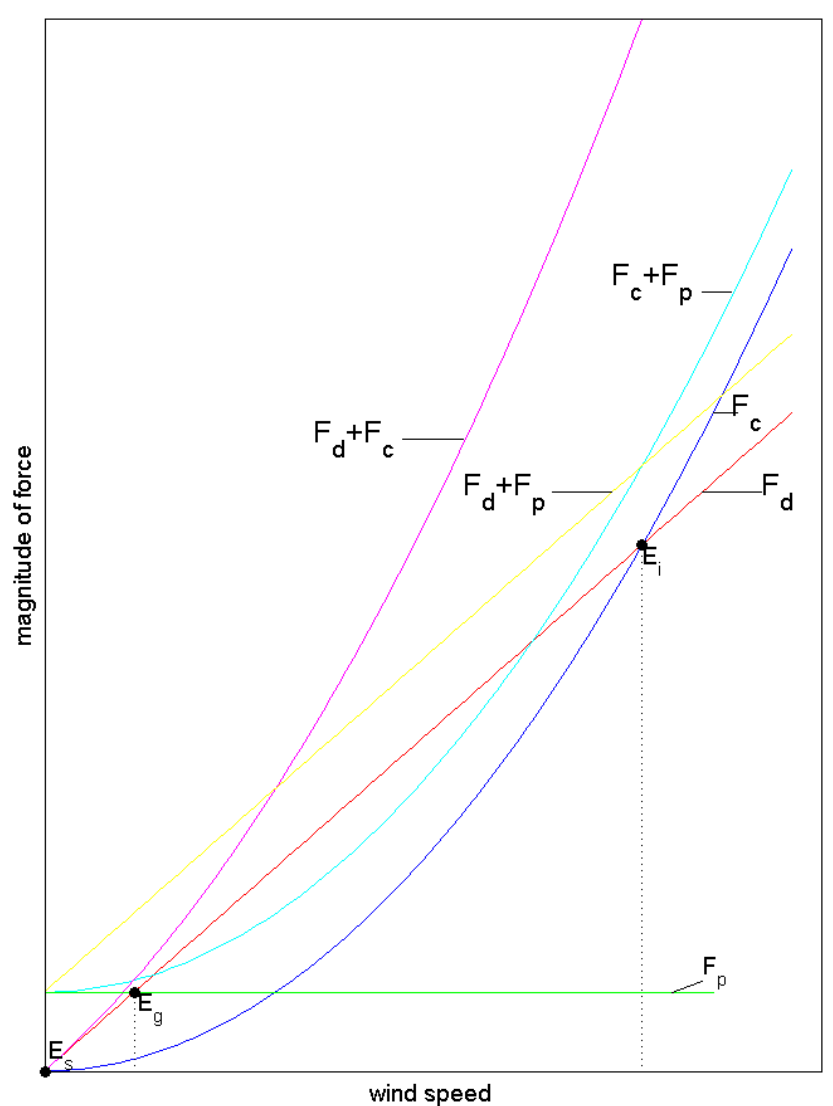

Figure 2. A wind-force relation diagram. The horizontal axis represents the wind speed and the vertical axis represents the magnitude of forces. Curve $F_{\mathrm{d}}$ represents the Coriolis force (deflection force of earth rotation), curve $F_{\mathrm{c}}$ the centrifugal force, curve $F_{\mathrm{p}}$ the pressure gradient forces, curve $F_{\mathrm{c}}+F_{\mathrm{p}}$ the sum of the centrifugal and the pressure gradient force, curve $F_{\mathrm{d}}+F_{\mathrm{p}}$ the sum of the Coriolis and the pressure gradient force and curve $F_{\mathrm{d}}+F_{\mathrm{c}}$ the sum of the Coriolis and the centrifugal force. The curves are calculated from the force magnitude function of wind speed. Point $E_{\mathrm{S}}$ represents the state of static wind, $E_{\mathrm{g}}$ the geostrophic wind and $E_{\mathrm{i}}$ the inertial wind.

Therefore, the Coriolis force $f V$ presents a straight line $F_{\mathrm{d}}$ passing through the origin, the centrifugal force $\frac{V^{2}}{R}$ presents a parabolic curve $F_{\mathrm{c}}$, and the pressure gradient force $\frac{1}{\rho} \frac{\partial p}{\partial n}$ presents a straight line $F_{\mathrm{p}}$ paralleled to the horizontal axis. If two forces are combined, the corresponding functions are also added to present a synthetic curve. For the normal high and the anomalous high, the wind has an anticyclonic trajectory. The Coriolis force at the right of the wind direction is balanced by the sum of the centrifugal and the pressure gradient force at the left, represented by curve $F_{\mathrm{c}}+F_{\mathrm{p}}$. For the normal low, the wind has a cyclonic trajectory. The pressure gradient force at the left of the wind direction is balanced by the sum of the Coriolis and the centrifugal force at the right, represented by curve $F_{\mathrm{d}}+F_{\mathrm{c}}$. For the anomalous low, the wind has an anticyclonic trajectory. The centrifugal force to the left of the wind direction is balanced by the sum of the Coriolis and the pressure gradient force, represented by curve $F_{\mathrm{d}}+F_{\mathrm{p}}$. Consequently, determined force magnitude can be found at a point on the corresponding curve given certain wind speed. When relevant forces are balanced with one another, the intersection of the curves is the balance state. Considering that the Coriolis force cannot be ignored for largescale air motion, there are two important initial balance states ( $E_{\mathrm{g}}$ and $E_{\mathrm{i}}$ in Fig. 2), representing the geostrophic and the inertial wind, respectively. The geostrophic wind is the most common balance state appearing in the free atmosphere due to the geostrophic feature of the Earth's atmosphere. The inertial wind is also an important balance state in the following discussion because it is related to mechanisms of the anomalous high and the anomalous low. In addition, static wind $\left(E_{\mathrm{S}}\right)$ should also be taken into account since it represents an initial balance without the influence of force such as the state near the Earth's surface before evolution of the wind.

Balance states are dynamic and wind continuously alternates between the balance and the evolution due to the variation of air pressure fields, wind fields and air trajectories. Solar radiation received by the Earth's surface is the major source of energy for air motion and its uneven distribution drives the average large-scale wind in the atmosphere. Meantime, it varies all the time because of the period fluctuation as well as changing weather and surface conditions. The uneven variation leads to local high or low pressure perturbation which is commonly observed in practice. Therefore the air pressure perturbation is the primary factor of wind evolution. When a local high or low pressure perturbation is generated, it will change the initial pressure gradient distribution since its magnitude is different from the surroundings. Hence a perturbed pressure gradient force is induced and the initial balance is destroyed. The magnitude of forces as well as the wind speed will evolve until another balance state is achieved through the adjustment of the pressure field and the wind field. The new balance state can be found at the intersection of curves for suitable balanced wind speed. The transformation from the initial balance state to the next balance state is just the evolution process of the wind. It is worthwhile noting that the curve of pressure gradient force will translate during the evolution since its magnitude is not related to wind speed.

\section{The normal high and the anomalous high}

\subsection{Normal high originating from the static wind}

The state of the original static wind is at $E_{\mathrm{s}}$ (Fig. 3). When a local high pressure perturbation $p^{\prime}$ is generated, the induced pressure gradient force accelerates the air outward from the center of the high pressure. Meanwhile the Coriolis force deflects the moving air to its right, leading to an anticyclonic trajectory as well as the centrifugal force. Although both the centrifugal force and the Coriolis force increase as the wind 


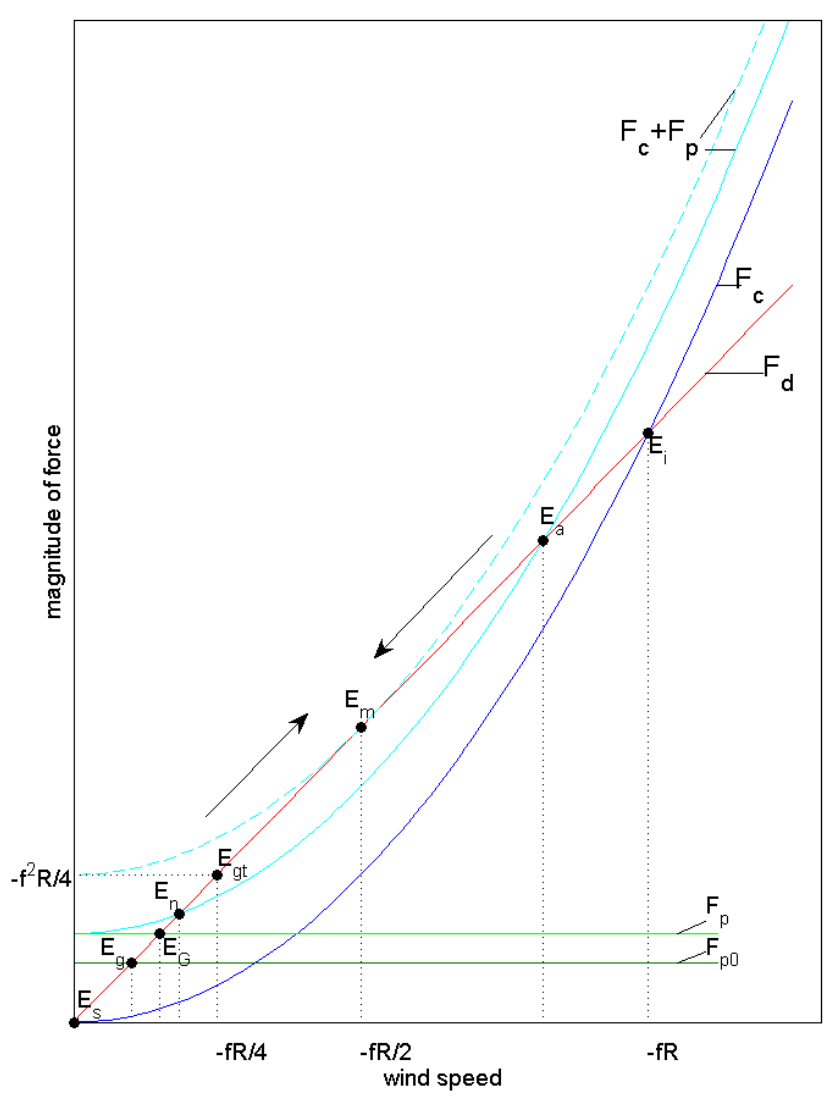

Figure 3. A diagram of the evolution of the normal high and the anomalous high. $F_{\mathrm{p}}$ represents the pressure gradient forces and $F_{\mathrm{p} 0}$ represents the pressure gradient force of the original geostrophic wind.

speed increases, the increase of the Coriolis force is faster than the centrifugal force when the wind speed is between 0 and $-f R / 2$. Therefore, the sum of the centrifugal force and the weakened pressure gradient force due to the flow crossing the isobars can be gradually balanced by the Coriolis force. The gradient wind of the normal high is then established and the wind arrives at a new state $E_{\mathrm{n}}$ with speed

$V=-\frac{f R}{2}-\left(\frac{f^{2} R^{2}}{4}-\frac{R}{\rho} \frac{\partial p}{\partial n}\right)^{1 / 2}$,

where $\frac{\partial p}{\partial n}$ is the pressure gradient when the balance is established $\left(\left|\frac{\partial p}{\partial n}\right|<\left|\frac{\partial p^{\prime}}{\partial n}\right|\right)$. The farther away from the high pressure center, the weaker the influence of the pressure gradient and the smaller the wind speed, i.e., $V \rightarrow 0$ when $R=\infty$.

It can be proved that the final pressure gradient force of the normal high originating from the static wind cannot exceed $-f^{2} R / 4$ and the corresponding wind speed cannot exceed $-f R / 2$. Because the Coriolis force is required to be balanced by the sum of the centrifugal force and the pressure gradient force, the pressure gradient force cannot exceed the difference of the Coriolis force and the centrifu- gal force when the balance is established. It can be seen in Fig. 3 that the maximum difference $-f^{2} R / 4$ is reached when the wind speed is equal to $-f R / 2$. In this situation, curve $F_{\mathrm{c}}+F_{\mathrm{p}}$ (the dotted curve in Fig. 3) touches curve $F_{\mathrm{d}}$ with only one intersect $E_{\mathrm{m}}$. If the final pressure gradient force exceeds $-f^{2} R / 4$, the balance cannot be established (the initial perturbed pressure gradient force may exceed $-f^{2} R / 4$ ). The state of the geostrophic wind with the maximum pressure gradient force $-f^{2} R / 4$ is at $E_{\mathrm{gt}}$ and its wind speed is $-f R / 4$, which is just half of the maximum wind speed of the normal high.

This type of normal high originating from the static wind can correspond to the flow of high pressure observed in the boundary layer of the atmosphere near the Earth's surface, where the geostrophic balance is not established and the friction cannot be ignored. In fact, this flow is a four-force process including friction in addition to the other three forces. The wind cannot flow strictly parallel to isobars but it diverges from the high pressure center, and thus it would not be maintained for a long time if there is no compensation from the upper level.

\subsection{Normal high originating from the geostrophic wind}

The state of the original geostrophic wind is at $E_{\mathrm{g}}$ (Fig. 3). When a local high pressure perturbation $p^{\prime}$ is generated, the sum of the original and the perturbed pressure gradient force is greater than the original Coriolis force. As a result, the air crosses isobars towards low pressure and is accelerated. Meanwhile it is deflected to the right by the Coriolis force, leading to an anticyclonic trajectory as well as the centrifugal force. The sum of the centrifugal force and the weakened pressure gradient force due to air crossing isobars can be gradually balanced by the Coriolis force. The gradient wind of the normal high is established and the wind arrives at a new state $E_{\mathrm{n}}$ with speed

$V=-\frac{f R}{2}-\left(\frac{f^{2} R^{2}}{4}-\frac{R}{\rho} \frac{\partial p}{\partial n}\right)^{1 / 2}$

where $\frac{\partial p}{\partial n}$ is the final pressure gradient when the balance is established $\left(\left|\frac{\partial p}{\partial n}\right|>\left|\frac{\partial p_{0}}{\partial n}\right|\right)$. The farther away from the high pressure center, the weaker the influence of the additional pressure gradient and the closer the wind speed approaches the original geostrophic wind speed (it is supposed that distant background pressure field of the original geostrophic wind is not influenced by the local pressure perturbation), i.e., $V \rightarrow V_{\mathrm{g}}$ when $R=\infty$. In addition, the wind speed of the geostrophic wind at $E_{\mathrm{G}}$ with the same pressure gradient force is smaller than that of the normal high at $E_{\mathrm{n}}$.

Similar to the normal high originating from the static wind, it can be proved that the pressure gradient force of the normal high originating from the geostrophic wind cannot exceed $-f^{2} R / 4$. Because the pressure gradient force of the gradient wind is larger than that of the original geostrophic wind, 
the pressure gradient force of the original geostrophic wind cannot exceed $-f^{2} R / 4$ either and the original geostrophic wind speed cannot exceed $-f R / 4$; that is to say, the state of the original geostrophic wind $E_{\mathrm{g}}$ should be between $E_{\mathrm{s}}$ and $E_{\mathrm{gt}}$.

This type of normal high originating from the geostrophic wind can correspond to ridges usually seen in high-altitude synoptic charts where the geostrophic balance dominates.

\subsection{Anomalous high originating from the inertial wind}

The state of the original inertial wind is at $E_{\mathrm{i}}$ (Fig. 3). When a local high pressure perturbation $p^{\prime}$ is generated, the corresponding pressure gradient force is induced and the air flows outward. Since the inertial wind speed is proportional to the radial distance as shown in Eq. (6), the outward flow transports the air with smaller momentum to the air with larger momentum along the path, resulting in a friction deceleration of the wind. Although both the centrifugal and the Coriolis force decrease as the wind speed decreases, the decrease of the centrifugal force is faster than the Coriolis force when the wind speed is between $-f R / 2$ and $-f R$. Therefore, the sum of the centrifugal force and the weakened pressure gradient force due to air crossing isobars can be gradually balanced by the Coriolis force. The gradient wind of the anomalous high is then established and the wind arrives at a new state point $E_{\mathrm{a}}$ with speed

$V=-\frac{f R}{2}+\left(\frac{f^{2} R^{2}}{4}-\frac{R}{\rho} \frac{\partial p}{\partial n}\right)^{1 / 2}$

where $\frac{\partial p}{\partial n}$ is the final pressure gradient when the balance is established $\left(\left|\frac{\partial p}{\partial n}\right|<\left|\frac{\partial p^{\prime}}{\partial n}\right|\right)$. Similar to the inertial wind, the farther away from the high pressure center, the greater the wind speed, i.e., $V \rightarrow \infty$ when $R=\infty$. Of course it is actually impossible for the curvature radius of the inertial wind or the anomalous high to approach infinity, so it is impossible for the wind speed to approach infinity.

Similarly, it can be proved that the final pressure gradient force of the anomalous high originating from the inertial wind cannot exceed $-f^{2} R / 4$. A minimum wind speed $-f R / 2$ for the anomalous high at the tangent point $E_{\mathrm{m}}$ for curve $F_{\mathrm{c}}+F_{\mathrm{p}}$ and curve $F_{\mathrm{d}}$ can be reached given the maximum pressure gradient force $-f^{2} R / 4$.

The anomalous high can be stable with respect to additional perturbations. Suppose that an anomalous high is at $E_{\mathrm{a}}$ in Fig. 3. When an additional high pressure perturbation $p^{\prime}$ is generated for the anomalous high, the perturbed pressure gradient force is induced and the air flows outwards to low pressure. The flow transports the air with smaller momentum to the air with larger momentum since the wind speed of the anomalous high increases with $R$, leading to friction which decreases the wind speed. Both the centrifugal and the Coriolis force decrease with the wind, but the centrifugal force decreases more quickly than the Coriolis force and thus a larger pressure gradient force is required to establish a new balance. Consequently, the Coriolis force can be gradually balanced by the sum of the centrifugal force and the strengthened pressure gradient force. A new gradient wind balance of the anomalous high is then established with a smaller wind speed than the original one at $E_{\mathrm{a}}$. When additional low pressure perturbation $p^{\prime \prime}$ is generated for the anomalous high, the discussion is similar as the high pressure perturbation $p^{\prime}$, a new balance of the anomalous high, can be established with a greater wind speed than the original one.

Since this type of anomalous high originates from the inertial wind, it would be more possible to be observed in places where inertial wind may appear. Regions of jet stream are likely cases because the wind can be more easily maintained when the pressure gradient force disappears and then turns to the inertial flow subject to the Coriolis force. For instance, high-level jet due to the thermal wind effect and the topographic jet may give more possibility to the occurrence of this type of the anomalous high.

\subsection{Anomalous high originating from the static wind or the geostrophic wind}

As discussed above, the final pressure gradient force for the normal high cannot exceed $-f^{2} R / 4$ but the pressure gradient force before the evolution may exceed $-f^{2} R / 4$. When the initial pressure gradient force is significantly greater than $-f^{2} R / 4$, the accelerating wind speed may approach $-f R / 2$ but the pressure gradient force is still larger than $-f^{2} R / 4$ at present though it is reduced due to air crossing isobars. Hence the balance state of the normal high is missed. The wind continues accelerating and exceeds $-f R / 2$, while the pressure gradient force is further reduced to be smaller than $-f^{2} R / 4$. Consequently, the wind arrives at a balance state in regime of the anomalous high.

This type of anomalous high originating from the static or the geostrophic wind requires large high pressure perturbation and sustained acceleration. A large local high pressure perturbation may occur more easily in the lower level because the heating of the atmosphere is mainly from the Earth's surface but the friction would retard the acceleration process. In the upper level of the atmosphere, the friction can be ignored but it is difficult to reach a large high pressure perturbation in a moment. Therefore, this type of the anomalous high is actually difficult to occur.

\subsection{Normal high originating from the inertial wind}

Similar to the anomalous high originating from the static or geostrophic wind discussed in Sect. 3.4, when the initial pressure gradient force for the inertial wind is significantly greater than $-f^{2} R / 4$, the decelerating wind speed may approach $-f R / 2$ but the balance state of the anomalous high is missed because the pressure gradient force is still larger than $-f^{2} R / 4$ at present. The wind speed continues decelerating 
and falls below $-f R / 2$ while the pressure gradient force is further reduced to be smaller than $-f^{2} R / 4$. Consequently, the wind arrives at a balance state in regime of the normal high.

It may be more difficult for this type of normal high originating from the inertial wind to occur, since it is more difficult for the inertial wind to appear than the static or the geostrophic wind.

\subsection{Transitions between the normal high and the anomalous high}

Similar to the mechanisms discussed in Sects. 3.4 and 3.5, when a high pressure perturbation is generated for the normal high, the initial pressure gradient force may be significantly greater than $-f^{2} R / 4$. The acceleration process may lead the wind to arrive at a balance state in regime of the anomalous high. When a high pressure perturbation is generated for the anomalous high, the decelerating wind may arrive at a balance state in regime of the normal high when the initial pressure gradient force is significantly greater than $-f^{2} R / 4$. This two-way transition between the normal high and the anomalous high is more possible for those with wind speed around $-f R / 2$ because in this situation, a large initial pressure gradient force is easier to achieve. This transition may be the primary mechanism of the anomalous high that could be observed in the Earth's atmosphere. Nevertheless, the anomalous high and the normal high originated by this mechanism are difficult to discriminate from each other since they have wind speeds close to each other, around $-f R / 2$. Krishnamurti et al. (2005) listed observed possible cases of anomalous wind and most of them had a wind speed slightly greater than the wind speed of the normal high.

\subsection{Summary}

Based on the above analysis, the evolution of gradient wind of the high pressure system is complex. There are three situations for formation mechanisms of the normal high and the anomalous high. The first is opposite processes from static or geostrophic wind for the normal high, and from the inertial wind for the anomalous high, respectively, indicated by the direction of the arrows in Fig. 3. The second is opposite processes from the static or geostrophic wind for the anomalous high, and from the inertial wind for the normal high, respectively, when the initial pressure gradient force is significantly larger than $-f^{2} R / 4$. The third is opposite transitions between the normal high and the anomalous high, especially for those with wind speeds around $-f R / 2$, which may be the most possible mechanism of the anomalous high in the Earth's atmosphere.

The normal high is driven by the pressure gradient force. The greater the pressure gradient force is, the greater the balanced wind speed is. In contrast, the pressure gradient force is not the power source of the anomalous high but it weak- ens the wind speed. The larger the pressure gradient force is, the smaller the balanced wind speed is. When the pressure gradient force is equal to $-f^{2} R / 4$, states of the normal high and the anomalous high fuse together at $E_{\mathrm{m}}$ with wind speed $-f R / 2$ which is the maximum wind speed for the normal high, while the minimum for the anomalous high. $-f R / 2$ is also just twice the geostrophic wind speed under the same pressure gradient force.

The only evidence to discriminate the anomalous high from the normal high is the wind speed. Previous studies usually focus on the upper atmosphere at 200 or $300 \mathrm{hPa}$ to search for the possible occurrence of the anomalous high (Angell, 1960; Mogil and Holle, 1972; Krishnamurti et al., 2005). However, we should pay more attention to the middle atmosphere rather than the upper atmosphere because the difference of wind speed of the anomalous high and the normal high is positively correlated with the air density. Suppose that at $500 \mathrm{hPa}$ and $45^{\circ} \mathrm{N}$, the wind flows parallel to the isobars with pressure gradient $1 \mathrm{hPa}(100 \mathrm{~km})^{-1}$. At a place $600 \mathrm{~km}$ away from the high pressure center, the wind speed is about $21.1 \mathrm{~m} \mathrm{~s}^{-1}$ for the normal high and is about $40.6 \mathrm{~m} \mathrm{~s}^{-1}$ for the anomalous high. By comparison, the geostrophic wind speed is about $13.9 \mathrm{~m} \mathrm{~s}^{-1}$, and the inertia wind speed is about $61.7 \mathrm{~m} \mathrm{~s}^{-1}$.

\section{The normal low and the anomalous low}

\subsection{Normal low originating from the static wind}

The state of the original static wind is at $E_{\mathrm{s}}$ (Fig. 4). When a local low pressure perturbation $p^{\prime}$ is generated, the induced pressure gradient force accelerates the air inward toward the center of the low pressure. Meanwhile the Coriolis force deflects the moving air to its right, leading to a cyclonic trajectory as well as the centrifugal force. The weakened pressure gradient force can be gradually balanced by the sum of the centrifugal and the Coriolis force. The gradient wind of the normal low is then established and the wind arrives at a new state point $E_{\mathrm{n}}$ with speed

$V=-\frac{f R}{2}+\left(\frac{f^{2} R^{2}}{4}-\frac{R}{\rho} \frac{\partial p}{\partial n}\right)^{1 / 2}$,

where $\frac{\partial p}{\partial n}$ is the pressure gradient when the balance is established $\left(\left|\frac{\partial p}{\partial n}\right|<\left|\frac{\partial p^{\prime}}{\partial n}\right|\right)$. The farther away from the low pressure center, the smaller the wind speed, i.e., $V \rightarrow 0$ when $R=\infty$.

This type of normal low can correspond to the flow of low pressure observed in the boundary layer. The wind would not flow strictly parallel to isobars but converge to the low pressure center, and it would not be maintained for a long time if there is no compensation flow. Another common case is the synoptic-scale hurricane at lower level. 


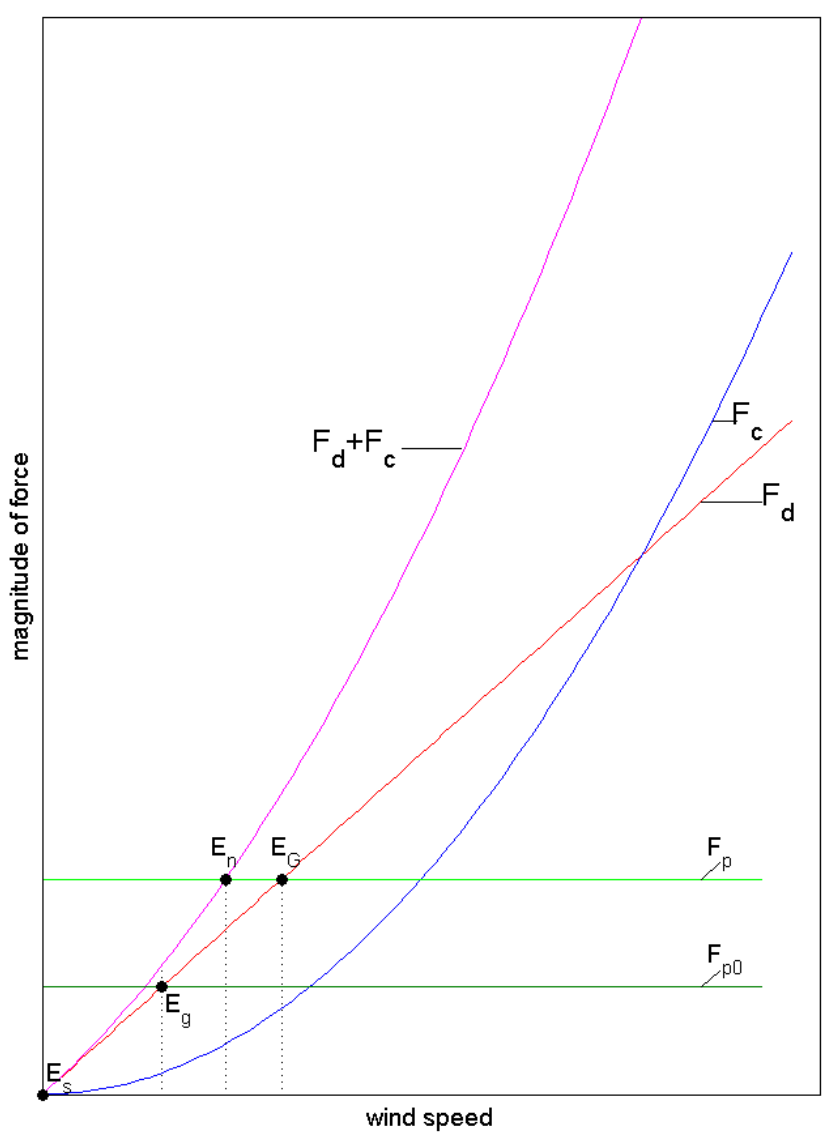

Figure 4. A diagram of the evolution of the normal low. $F_{\mathrm{p}}$ represents the pressure gradient forces and $F_{\mathrm{p} 0}$ represents the pressure gradient force of the original geostrophic wind.

\subsection{Normal low originating from the geostrophic wind}

The state of the original geostrophic wind is at $E_{\mathrm{g}}$ (Fig. 4). When a local low pressure perturbation $p^{\prime}$ is generated, the sum of the original and the perturbed pressure gradient force is greater than the original Coriolis force. As a result, the air crosses isobars towards low pressure and is accelerated. Meanwhile it is deflected to the right by the Coriolis force, leading to a cyclonic trajectory as well as the centrifugal force. The weakened pressure gradient force can be balanced by the sum of the centrifugal and the Coriolis force. The gradient wind of the normal low is then established and the wind arrives at a new state point $E_{\mathrm{n}}$ with speed

$$
V=-\frac{f R}{2}+\left(\frac{f^{2} R^{2}}{4}-\frac{R}{\rho} \frac{\partial p}{\partial n}\right)^{1 / 2}
$$

where $\frac{\partial p}{\partial n}$ is the pressure gradient when the balance is established $\left(\left|\frac{\partial p}{\partial n}\right|>\left|\frac{\partial p_{0}}{\partial n}\right|\right)$. The farther away from the low pressure center, the closer the wind speed approaches the original geostrophic wind speed (it is supposed that the background pressure field of the geostrophic wind is not influenced by

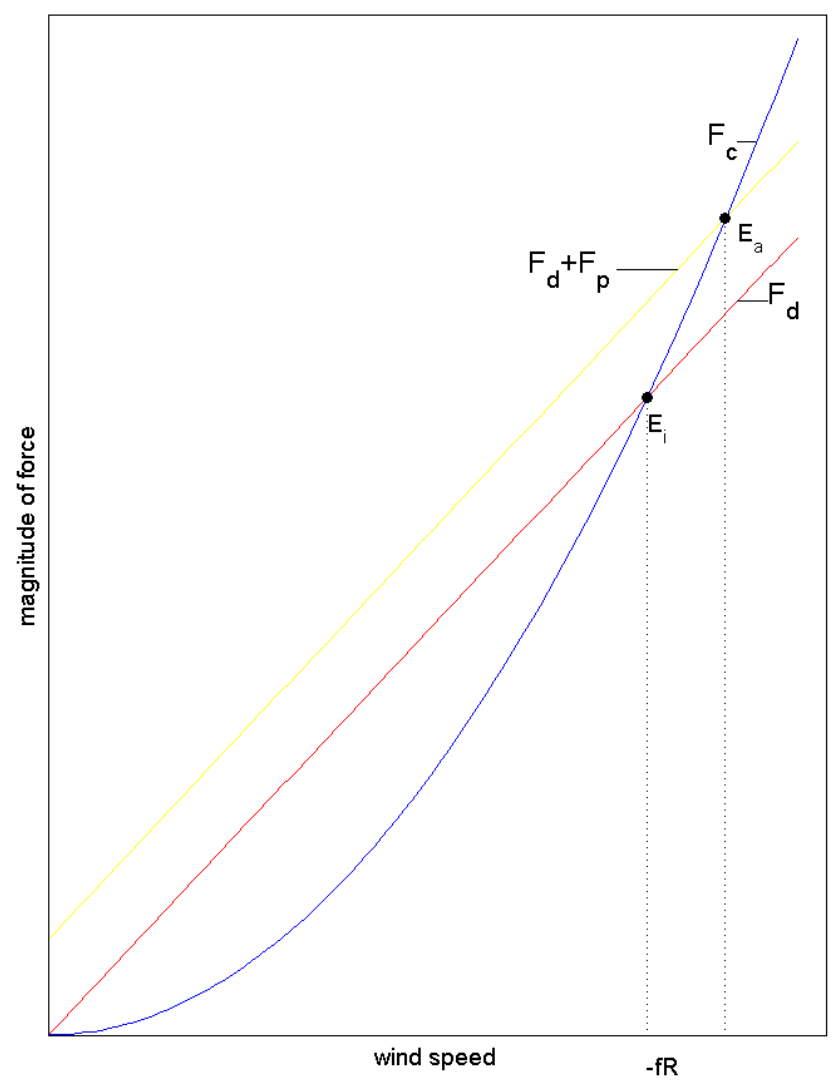

Figure 5. A diagram of the evolution of the anomalous low.

the local pressure perturbation), i.e., $V \rightarrow V_{\mathrm{g}}$ when $R=\infty$. In addition, it can be seen in Fig. 4 that the wind speed of the geostrophic wind at $E_{\mathrm{G}}$ with the same pressure gradient force is greater than the normal low.

This type of normal low originating from the geostrophic wind can correspond to troughs usually seen in high-altitude synoptic charts where the geostrophic balance dominates.

\subsection{Anomalous low originating from the inertial wind}

The state of the original inertial wind is at $E_{\mathrm{i}}$ (Fig. 5). When a local low pressure perturbation $p^{\prime}$ is generated, the corresponding pressure gradient force is induced and the air flows inward. The flow transports the air with larger momentum to the air with smaller momentum along the path since the inertial wind speed is proportional to the radial distance, resulting in acceleration of the wind speed. Because the Coriolis force is smaller than the centrifugal force when the wind speed is more than $-f R$, the sum of the Coriolis force and the weakened pressure gradient force can be balanced by the centrifugal force. The gradient wind of the anomalous low is then established and the wind arrives at a new state point $E_{\mathrm{a}}$ with speed

$$
V=-\frac{f R}{2}+\left(\frac{f^{2} R^{2}}{4}-\frac{R}{\rho} \frac{\partial p}{\partial n}\right)^{1 / 2}
$$


where $\frac{\partial p}{\partial n}$ is the pressure gradient when the balance is established $\left(\left|\frac{\partial p}{\partial n}\right|<\left|\frac{\partial p^{\prime}}{\partial n}\right|\right)$. The farther away from the low pressure center, the closer the wind speed approaches the inertial wind speed. The wind speed of the anomalous low is greater than the original inertial wind.

The anomalous low can be stable with respect to additional perturbations. Suppose that an anomalous low is at $E_{\mathrm{a}}$ in Fig. 5. When additional low pressure perturbation $p^{\prime}$ is generated for the anomalous low, the additional pressure gradient force is induced and the air flows inwards to the low pressure. The flow transports the air with larger momentum to the air with smaller momentum, leading to an acceleration of the wind speed. Both the centrifugal force and the Coriolis force increase with the wind, but the centrifugal force increases more quickly than the Coriolis force and thus a larger pressure gradient force is required to establish a new balance. Consequently, the centrifugal force can be gradually balanced by the sum of the Coriolis force and the strengthened pressure gradient force. Therefore, a new gradient wind balance of the anomalous low is then established with a larger wind speed than the original anomalous low. When additional high pressure perturbation $p^{\prime \prime}$ is generated for the anomalous high, the discussion is similar as for the situation of low pressure perturbation; a new balance of the anomalous low can be established with a smaller wind speed than the original anomalous low.

It would be possible to observe the anomalous low where the inertial wind may appear. A possible case is over the Gulf of Tehuantepec, Mexico, where researchers studied the southward gap wind through the Chivela Pass (Parmenter, 1970; Flores-Vidal et al., 2014). This gap outflow extends several hundreds of kilometers southward over the eastern Pacific and turns anticyclonically. Some scholars declared that this anticyclonic outflow is the inertial wind (Clarke, 1988; Schultz et al., 1997). They proposed that the curvature radius for trajectories of this gap wind matched that for balanced inertial flow. Steenburgh et al. (2006) studied the structure and evolution of this gap outflow using a high-resolution simulation of a numerical model as well as the observation. They found the cross-flow pressure gradient produced a fanlike wind pattern rather than a narrow jet. In addition, they found that wind trajectories to the west of the outflow jet showed stronger anticyclonic flow curvature than predicted by inertial balance. Because the cross-flow pressure gradient points to the west, this accords with the appearance of the anomalous low to the west of the outflow jet with its curvature larger than that of the inertial wind.

\subsection{Summary}

Based on the above analysis, the normal low and the anomalous low are balance processes from the static wind or geostrophic wind, and from the inertial wind, respectively. Both the normal low and the anomalous low are driven by the pressure gradient force. The greater the pressure gradient force, the greater the balanced wind speed. For both types, the wind speed has no limit.

Suppose that the air pressure gradient is $1 \mathrm{hPa}(100 \mathrm{~km})^{-1}$ at $15^{\circ} \mathrm{N}$. At a place $600 \mathrm{~km}$ away from the low pressure center above the sea surface, the wind speed is about $13.8 \mathrm{~m} \mathrm{~s}^{-1}$ for the normal low and is about $36.3 \mathrm{~m} \mathrm{~s}^{-1}$ for the anomalous low. By comparison in the same condition, the geostrophic wind speed is about $22.1 \mathrm{~m} \mathrm{~s}^{-1}$, and the inertia wind speed is about $22.6 \mathrm{~m} \mathrm{~s}^{-1}$. The actual wind speed would be smaller than these values because of the friction. This situation and these results are generally in agreement with the gap wind over the Gulf of Tehuantepec in previous studies (Schultz et al., 1997; Steenburgh et al., 1998).

\section{Conclusions and prospects}

This manuscript gives a discussion on the gradient wind balance subject to the Coriolis, the centrifugal and the pressure gradient forces. Formation mechanisms and possible existence of the normal high, the normal low, the anomalous high and the anomalous low are analyzed in detail. The results give reasonable explanation for both the anomalous high and the anomalous low, both of which are usually considered to be pure mathematical solutions and are assigned no physical meaning in classical literature of atmospheric sciences.

The non-uniform distribution of received solar insolation results in the temperature difference and thus makes for the widespread geostrophic wind. Due to the geostrophic feature of the atmosphere, the normal high and the normal low are commonly observed, while this is not the case for the anomalous high and the anomalous low because the inertial wind influences the Earth's atmosphere little. Compared with the anomalous low, the anomalous high may occur more frequently since it could also originate from the normal high. Although there has been no conclusive evidence up to now to prove the observation of anomalous types of the gradient wind, it is more possible to observe them in jet regions where the inertial wind and the normal high of large wind speed are easier to appear. More attention should be paid to these regions in order to prove possible occurrence of the anomalous gradient wind. Moreover, for other planets where the atmospheric condition might be conducive to the prevailing of the inertial wind, the anomalous high and the anomalous low might be the common gradient wind. For example, a planet revolves around a distance star, and thus has insignificant temperature differences between latitudes.

Through the deep discussion on the gradient wind including the anomalous high and anomalous low, motion and evolution principles of the Earth's atmosphere can be comprehensively understood. Beyond the theoretical significance, the gradient wind may serve as a better estimate of a wind field than the geostrophic wind (Dutton, 1986; Holton, 2004). The gradient wind can be used as a background or reference 
state in analytic or numerical modeling (Hodyss and Nolan, 2007). Numerical models generally exclude the occurrence of the anomalous gradient wind through geostrophic balance and physical constraints, but this may cause failure forecasting for cases (Krishnamurti et al., 2005). Therefore, the numerical models would be improved with consideration of the anomalous gradient wind which does have physical meaning and may occur in the atmosphere.

Acknowledgements. The author is grateful to the referees and editors for their constructive criticism and suggestions regarding an earlier version of this paper. This study was supported by Young Scientists Fund of the National Natural Science Foundation of China (41305031), Young Scientists Fund of the Natural Science Foundation of Jiangsu Province of China (BK2012466), Special Scientific Research Fund of Meteorological Public Welfare Profession of China (GYHY201306078), and the Priority Academic Program Development (PAPD) of Jiangsu Higher Education Institution.

The topical editor V. Kotroni thanks two anonymous referees for help in evaluating this paper.

\section{References}

Alaka, M. A.: The occurrence of anomalous winds and their significance, Mon. Weather Rev., 89, 482-494, 1961.

Angell, J. K.: An analysis of operational 300-MB transosonde flights from Japan in 1957-58, J. Atmos. Sci., 17, 20-35, 1960.

Brill, K. F.: Revisiting an old concept: the gradient wind, Mon. Weather Rev., 142, 1460-1471, 2014.

Byers, H. R.: General Meteorology, McGraw-Hill, New York, USA, 166-169, 1974.

Clarke, A. J.: Inertial wind path and sea surface temperature patterns near the Gulf of Tehuantepec and Gulf of Papagayo, J. Geophys. Res., 93, 15491-15501, 1988.

Dutton, J. A.: The Ceaseless Wind, Dover Publications Inc., New York, USA, p. 311, 1986.

Flores-Vidal, X., Durazo, R., Zavala-Sanson, L., Flament, P., Chavanne, C., Ocampo-Torres, F. J., and Reyes-Hernandez, C.: Evidence of inertially generated coastal-trapped waves in the eastern tropical Pacific, J. Geophys. Res.-Oceans, 119, 3121-3133, 2014.
Fultz, D.: Quantitative nondimensional properties of the gradient wind, J. Atmos. Sci., 48, 869-875, 1991.

Godske, C. L., Bergeron, T., Bjerknes, J., and Bundgaard, R. C.: Dynamic Meteorology and Weather Forecasting, Am. Meteorol. Soc., Boston, USA, 369-376, 1957.

Haltiner, G. J. and Martin, F. L.: Dynamical and Physical Meteorology, McGraw-Hill, New York, USA, 183-190, 1957.

Hess, S. L.: Introduction to Theoretical Meteorology, Krieger Pub. Co., Malabar, FL, USA, 180-185, 1959.

Hodyss, D. and Nolan, D. S.: Linear anelastic equations for atmospheric vortices, J. Atmos. Sci., 64, 2947-2959, 2007.

Holton, J. R.: An Introduction to Dynamic Meteorology, Academic Press, San Diego, USA, 67-69, 1992.

Holton, J. R.: An Introduction to Dynamic Meteorology, Elsevier Academic Press, p. 65, 2004.

Humphreys, W. J.: Physics of the Air, McGraw-Hill, New York, USA, 119-126, 1940.

Jacobson, M. Z.: Fundamentals of Atmospheric Modeling, Cambridge University Press, Cambridge, UK, 114-117, 2005.

Knox, J. A. and Ohmann, P. R.: Iterative solutions of the gradient wind equation, Comput. Geosci., 32, 656-662, 2006.

Krishnamurti, T. N., Cunningham, P., and Rajendran, K.: Anomalous gradient winds in the subtropical jet stream and interpretations of forecast failures, Meteorol. Atmos. Phys., 88, 237-250, 2005.

Mogil, H. M. and Holle, R. L.: Anomalous gradient winds existence and implications, Mon. Weather Rev., 100, 709-716, 1972.

Panofsky, H. A.: Introduction to Dynamic Meteorology, Pennsylvania State University, University Park, USA, 76-81, 1956.

Parmenter, F. C.: Picture of the month: A "Tehuantepecer", Mon. Weather Rev., 98, p. 479, 1970.

Schultz, D. M., Bracken, W. E., Bosart, L. F., Hakim, G. J., Bedrick, M. A., Dickinson, M. J.m and Tyle, K. R.: The 1993 Superstorm cold surge: Frontal structure, gap flow, and tropical impact, Mon. Weather Rev., 125, 5-39, 1997.

Steenburgh, W. J., Schultz, D. M., and Colle, B. A.: The Structure and Evolution of Gap Outflow over the Gulf of Tehuantepec, Mexico, Mon. Weather Rev., 126, 2673-2691, 1998.

Wallace, J. M. and Hobbs, P. V.: Atmospheric Science: An Introductory Survey, Elsevier Academic Press, Amsterdam, the Netherlands, 379-383, 2006.

Willett, H. C. and Sanders, F.: Descriptive Meteorology, Academic Press, New York, USA, 128-132, 1959.

Willoughby, H. E.: The Golden Radius in Balanced Atmospheric Flows, Mon. Weather Rev., 139, 1164-1168, 2011. 